\title{
LA ENSEÑANZA DE LA FILOSOFÍA DESDE UNA PERSPECTIVA DE FILOSOFÍA CON NIÑOS
}

\author{
The TEACHING OF PHILOSOPHY THROUGH THE \\ PERSPECTIVE OF PHILOSOPHY WITH CHILDREN.
}

\author{
MAIRA FRUTOS \\ Universidad Nacional de Rosario - CONICET \\ mairafrutos@hotmail.com
}

RECIBIDO: 27 DE SEPTIEMBRE DE 2019

ACEPTADO: 22 DE DICIEMBRE DE 2019

Resumen: En el siguiente escrito nos proponemos pensar la enseñanza de la filosofía a partir de la experiencia obtenida a través de filosofía con niños. Para ello consideramos necesario abordar el trabajo de la siguiente manera. Primero definiremos qué entendemos por filosofía, luego haremos lo mismo con el concepto de enseñanza; y por último intentaremos proponer cómo entendemos la enseñanza de la filosofía, a partir de las herramientas obtenidas en filosofía con niños. Consideramos que la Filosofía es una disciplina cuya enseñanza puede representar un problema, pero no cualquier tipo de problema. Sino, que este es, a su vez, un problema filosófico y también, un problema político. Entonces, recurrimos a $\mathrm{FcN}$ para adoptar estrategias que nos ayuden a transmitir la filosofía como práctica de la mejor forma posible. Generalmente, comenzamos a indagar y a reflexionar acerca de la práctica docente, con suerte, una vez que estamos próximos a ingresar al aula. Otras veces, cuando nos ha tocado estar frente a estudiantes y no nos esperábamos esa situación.Si realmente nos interesa la enseñanza de la filosofía de manera que no se convierta en una mera transmisión de un conocimiento académico, sino que buscamos que los estudiantes se apropien de la filosofía y la tomen como una forma de vida. Si buscamos que la filosofía les permita reflexionar acerca de las experiencias que les tocan vivir, y que a partir de esa reflexión crítica puedan construir sentido. Si pretendemos que en las clases de filosofía surja el debate, el diálogo atento, se construyan comunidades de investigación. Entonces, nuestra forma de hacer filosofía en el aula, debe estar guiada, debe estar nutrida por $\mathrm{FcN}$. 
Palabras claves: filosofía, enseñanza, filosofía con niños, enseñanza filosófica.

\begin{abstract}
The teaching of Philosophy by the perspective of Philosophy with children. The purpose of the following selection is to consider the teaching of philosophy taking as a starting point the experience it occurred with Philosophy with Children $(\mathrm{PwC})$. What follows it's a description of how the text is structured. First, you will find the definition of the concept of philosophy. Second, you will read about the concept of teaching. At last, there will be theories and tools to put into practice in the teaching of Philosophy for Children. The process of teaching philosophy is intended to be though a problem in which a philosophical or political issue is stated. As a consequence, $\mathrm{PwC}$ plays an important role in providing students with strategies that may help the facilitator to transmit philosophical concept and related items such as practice.

The focus of teaching philosophy should be from the perspective of providing students with tools that helps them to develop abilities for life. Taking in consideration this, students would have the opportunity of building, creating and reflecting on their own experience and reality. It is important that teachers have an insight on philosophy with children so as to foster critical thinking in their students and improve their own teaching practice. Taking into consideration this never ending process of learning and teaching, children are the present and the hoping future.
\end{abstract}

Key words: philosophy, teaching, philosophy with children, philosophical teaching.

\title{
Introducción
}

En el siguiente escrito nos proponemos pensar la enseñanza de la filosofía a partir de la experiencia obtenida a través de filosofía con niños. Para ello consideramos necesario abordar el trabajo de la siguiente manera. Primero definiremos qué entendemos por filosofía, luego haremos lo mismo con el concepto de enseñanza; y por último intentaremos proponer cómo entendemos la enseñanza de la filosofía, a partir de las herramientas obtenidas en filosofía con niños.

Consideramos que la Filosofía es una disciplina cuya enseñanza puede representar un problema, pero no cualquier tipo de problema. 
Sino, que este es, a su vez, un problema filosófico y también, un problema político. Entonces, recurrimos a $\mathrm{FcN}$ para adoptar estrategias que nos ayuden a transmitir la filosofía como práctica de la mejor forma posible.

Generalmente, comenzamos a indagar y a reflexionar acerca de la práctica docente, con suerte, una vez que estamos próximos a ingresar al aula. Otras veces, cuando nos ha tocado estar frente a estudiantes y no nos esperábamos esa situación.

Si realmente nos interesa la enseñanza de la filosofía de manera que no se convierta en una mera transmisión de un conocimiento académico, sino que buscamos que los estudiantes se apropien de la filosofía y la tomen como una forma de vida. Si buscamos que la filosofía les permita reflexionar acerca de las experiencias que les tocan vivir, y que a partir de esa reflexión crítica puedan construir sentido. Si pretendemos que en las clases de filosofía surja el debate, el diálogo atento, se construyan comunidades de investigación. Entonces, nuestra forma de hacer filosofía en el aula, debe estar guiada, debe estar nutrida por $\mathrm{FcN}$.

Kohan y Cerletti, en el libro La filosofía en la escuela, nos dicen que "cuando una práctica pedagógica sigue el camino del análisis y las fundamentaciones, de la búsqueda y explicitación de supuestos, del descubrimiento de diversos marcos de relaciones en ciertas temáticas y situaciones de la puesta en cuestión de valores subyacentes, a partir de la criticidad y la creatividad, la calificamos de filosofía"

Sin embargo, si bien coincidimos con ambos autores, nos vemos en la necesidad de aclarar que consideramos que la filosofía se puede definir de muchas formas. Por lo tanto, citaremos otros autores que también intentaron definir qué entendían por filosofía, para seguir

${ }^{1}$ CERLETTI, A., KOHAN, W., La Filosofía en la escuela, La UBA y los Profesores Secundarios. Oficina de publicaciones de C. B. C., Buenos Aires, 1996, P. 137 
ampliando esta construcción del concepto que nos proponemos hacer.

Por ejemplo, García Morente, dirá que para definir qué es la filosofía, primero se debe tener de ella una vivencia. En palabras del autor "se debe ingresar a la filosofía como en una selva, para explorarla""

A esta idea puede sumarse lo propuesto por Heidegger, quien sostenía que preguntarse ‘qué es la filosofía?' sería intentar penetrar en la filosofía, demorarse en ella; preguntarse qué es la filosofía, sería filosofar. ${ }^{3}$

Pensamos en la filosofía como una forma de vivir, como una forma de ser en el mundo. Pero también, consideramos que la filosofía es búsqueda e interrogación. Para Karl Jaspers, filosofía quiere decir ir de camino. Según este autor, el sentido de la palabra filosofía es búsqueda de la verdad. Las preguntas resultan más esenciales que las respuestas, y toda respuesta se convierte, a su vez, en una nueva pregunta. ${ }^{4}$

Kohan y Cerletti ${ }^{5}$, aseguran que el carácter cuestionador y problemático de la pregunta señala el camino de la filosofía que está más ligado al cuestionamiento que a las certezas. Es "un

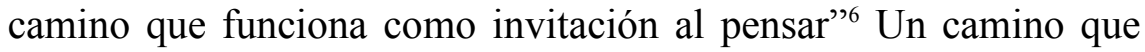
invita al diálogo, a un diálogo que puede ser con uno mismo, pero que también es un diálogo con otro, y con otros.

Por último, podríamos decir que la filosofía es un ejercicio político. Es decir, es un ejercicio de liberación. Es un acto que produce un cambio en las personas, pero también, produce un cambio en sus

2 GARCÍA MORENTE, M.: op.cit. P.5

${ }^{3}$ Heidegger, M., ¿Qué es la filosofia?, Barcelona, Herder, 2004; P. 51

${ }^{4}$ JASPERS, K., La Filosofía desde el punto de vista de la existencia, Fondo de cultura económica, México, 1953. P.25s

${ }^{5}$ CERLETTI, A., KOHAN, W., op.cit. P. 82

${ }^{6}$ CERLETTI, A., KOHAN, W., op.cit. P. 83 
mundos y en la forma que tienen de entender e interpretar esos mundos. La filosofía es un acto, una práctica, que permite reflexionar críticamente acerca de la sociedad, la democracia, la construcción ciudadana y las tensiones de poder que se dan en la convivencia cotidiana.

Ahora bien, teniendo en cuenta que no existe una única forma de entender la filosofía, ¿a qué nos referimos cuando hablamos de “enseñanza de la filosofía”? ¿Es posible enseñar filosofía? ¿Cómo? ¿Se enseña filosofía, o es mejor enseñar a hacer filosofía?

Algunos pueden sostener, como nos dice Federico Uicich, que según la concepción que se tenga de la filosofía, se podrá entender su enseñanza "como una actividad propiamente filosófica o como un mero trabajo que nos permite conseguir un salario" Desde nuestro lugar consideramos que la filosofía es una forma de vivir, es una herramienta, es una disciplina, es un saber, y como cualquier saber, puede ser transmitido. Nuestra pregunta en todo caso sería, ¿para qué transmitir filosofía? ¿Cuál es el interés que lleva a los profesores y profesoras a enseñar, a transmitir, el filosofar?

Consideramos que cuando pensamos en la enseñanza de la filosofía, no lo hacemos con la intención de transmitir la filosofía como un saber enciclopédico; sino, como sostiene Cerletti, lo hacemos para "construir una didáctica filosófica cuya filosofía subyacente sea una filosofía del encuentro, del azar y del acontecimiento, que posibilite aprendizajes filosóficos" ${ }^{\text {" }}$

$\mathrm{O}$ dicho de otra manera, aprendizajes filosóficos de manera filosófica. Es decir, una enseñanza que les permita a los estudiantes reflexionar sobre sus propias experiencias de manera que no sean

${ }^{7}$ UICIH, F., "Las decisiones filosóficas detrás de las decisiones didácticas: consejos para repensar la planificación de clases de filosofía", en CERLETTI, A., COULÓ, A., (2015), Didácticas de Filosofía. Entre enseñar y aprender a filosofar, Buenos Aires, Ediciones Novedades Educativas, P. 154)

${ }_{8}^{8}$ CERLETTI, A., COULÓ, A., (2015), Didácticas de Filosofia. Entre enseñar y aprender a filosofar, Buenos Aires, Ediciones Novedades Educativas, P.18 
receptores pasivos de lo que se le transmite. Sino, que se los motive a realizar el ejercicio de pensamiento por ellos mismos.

Cerletti nos dirá que "la posibilidad de que algo sea, se debe a la posibilidad de la interrupción; y la consecuente generación posterior de algo nuevo" Es decir, según el autor, para que haya aprendizaje, y en nuestro caso diríamos, aprendizaje filosófico, la continuidad de los contenidos y las prácticas instituidas deben verse desbordadas por la irrupción de algo que iría más allá de lo prescripto. Más allá de lo que habitualmente se concibe como enseñanza tradicional y meramente expositiva.

Coincidimos con Cerletti en que, para que se lleve a cabo una modificación entre lo que se transmite y lo que se interpreta de esa transmisión, se debe posibilitar una transformación. Debe acontecer una disrupción de la unidireccionalidad del discurso dominante, debe manifestarse un nuevo proceso dialógico donde acontezca un sujeto colectivo, y donde se cuestione y se reacomoden los saberes vigentes en una situación. ${ }^{10}$

Resignificar los lugares de quienes enseñan, quienes aprenden, y también, resignificar los lugares de lo enseñado y de lo aprendido. Posibilitando un encuentro que transforme a quienes participan en el encuentro y a los saberes puestos en juego.

Para ello, nos resulta necesario pensar críticamente en la relación que existe entre el profesor o profesora y los estudiantes. Y preguntarnos ¿Cuál es el rol de los profesores? ¿Con qué herramientas cuentan? ¿Qué estrategias pueden construir? ¿Se piensa en los estudiantes? ¿Cuándo? ¿De qué forma?

Hablamos de profesores, docentes, que cumplirían la función de acompañar, de transmitir, de preparar a los estudiantes para un aprendizaje que los haga libres, con habilidades que les permitan

${ }^{9}$ CERLETTI, A. op.cit. P. 17
${ }^{10}$ CERLETTI, A., op.cit. P. 24 
ser flexibles, críticos, creativos, cuidantes, miembros de una sociedad.

Rancière nos habla de un maestro que, queriendo emancipar, debe preguntar a la manera de los hombres y no de los sabios, para ser instruido y no para instruir. Es decir, aquél que pretende emancipar, debe preguntar como aquél que ignora la respuesta, pero guiando indirectamente la inteligencia del estudiante. ${ }^{11}$ Pues esa actitud deja al estudiante buscar, crear sus respuestas, pero sin descuidarlo, sin dejarlo solo en esa búsqueda. Sino que mantiene al que busca en su rumbo, rumbo en el que está solo en su búsqueda, pero que nunca deja de buscar.

Ranciére también dice que para emancipar hay que estar emancipado. Es decir, volverse turista de sí mismo. Interrogarse sobre lo que conoce y sobre lo que hace con aquello que conoce. Se trata de una búsqueda, volver y revolver las palabras, los pensamientos. Analizar la atención que se pone mientras se busca. $^{12}$

En el artículo titulado La praxis filosófica en el marco institucional $^{13}$, encontramos que, las autoras citan a Onfray para pensar la cuestión de la enseñanza de la filosofía. Pero lo transcribo porque creo que resulta interesante para pensar la función de los docentes en general.

Los alumnos se entusiasmarían más si eventualmente la materia estuviese pensada para ellos (...) La incurable melancolía en la que se mueven los alumnos tiene su raíz en la complacencia casi generalizada de los pensadores o educadores en dirección a la negatividad y la sospecha, en el silencio que ofrecen como única respuesta a la pregunta que los estudiantes hacen y se hacen sobre el

${ }^{11}$ RANCIÉRE, J., "El maestro ignorante”, Editorial Laertes, Barcelona, (2003), P. 20

${ }^{12}$ RANCIÉRE, J., op.cit. P.23

${ }^{13}$ CERLETTI, A. (Comp.), La enseñanza de la filosofia en perspectiva, Ed. Eudeba, Buenos Aires, (2009), P. 140 
sentido de la vida, en la falta de alternativa que encuentran frente al nihilismo de la época, en la incapacidad de captar la naturaleza de la realidad que es la suya, en el pesimismo que los rodea cuando expresan entusiasmo, pasión, deseos.(...) Se supone que deben armar hombres libres, pero fabrican esclavos ${ }^{14}$

Creo que la tarea de los docentes debe incluir pensar, no solo en lo que pretende enseñar, y en cómo enseñarlo, sino también, debe tener en cuenta a quién va a enseñárselo y dónde. En general, no se tiene presente a los estudiantes. En general, cuando se planifica, o se realizan currículums institucionales, no se tiene en cuenta al alumnado. Pero soy de los que consideran que, en el momento de planificar, deberíamos tener presente al grupo con el cual vamos a interactuar en las escuelas. Y no sólo a ellos con sus inquietudes y tiempos, sino también sus contextos.

Juan Nesprías, en uno de sus artículos nos dice algo en relación a la enseñanza de la filosofía, pero me resulta oportuno citarlo porque su interpretación, nos serviría para pensar la tarea de enseñar en cualquier disciplina.

la tarea de pensar la enseñanza filosófica en contextos "desfavorables", obliga a ampliar la mirada sobre las condiciones en las que se enseña la filosofía, y a vincular lo que ocurre en el aula, con lo que ocurre fuera de ella ${ }^{15}$

Pensar en las realidades de los estudiantes, nos lleva a pensar en la práctica de los profesores. Como si fuese un espiral que, al girar, va tocando puntos en diferentes lugares, pero que todos pertenecen a

${ }^{14}$ Onfray, M., "El deseo de ser un volcán”, en Cerletti, A. (Comp.), 2009, La enseñanza de la filosofía en perspectiva, Buenos Aires, Ed. Eudeba, P. 141.

${ }^{15}$ NESPRÍAS, J., "Enseñanza de la filosofía y contextos 'difíciles': límites, posibilidades, desafíos (una aproximación desde El maestro ignorante, de Jacque Ranciére", en Cerletti, A. (Comp.), (2009), La enseñanza de la filosofía en perspectiva, Buenos Aires, Ed. Eudeba, P.91 
una misma línea. Ahora bien, cuando se tiene presente que no todas las realidades son iguales, y que no todos los chicos tienen la misma situación económica, por poner algún ejemplo, lo que el autor nos propone es pensar la enseñanza desde la igualdad como inicio y no como fin. Es decir, siguiendo a Ranciére, nos dice que se debe partir de la idea de igualdad de inteligencias para romper ciertos estereotipos, ciertos paradigmas. De esta forma se cambia la perspectiva desde la cual se observa a los estudiantes. Ya no se los verá como aquellos que necesitan, que carecen, sino que se los verá como aquellos que tienen que comenzar su búsqueda, que tienen que hacer su camino, pero que todos tienen inteligencia para hacerlo.

Esto último nos hace pensar en otro factor sobre el que no se repara habitualmente, pero que Flavia Terigi nos invita a pensar en ello. En una de sus conferencias se puede leer que pretende cuestionar una idea muy fuerte que suele estructurar la enseñanza actual, según la cual, "para lograr aprendizajes equivalentes, se requieren enseñanzas similares" 16

La autora asegura que, en el aula, los docentes dicen algo del orden de la enseñanza con la expectativa de que todos lo escuchen, que todos entiendan lo mismo, y que lo aprendan de la misma forma que él lo previó cuando realizó la planificación. ${ }^{17}$

Sin embargo, según Terigi, se deben tener en cuenta diferentes aspectos; por ejemplo, no solo deberían pensar en que los chicos estén en la escuela, completen los niveles educativos, y aprendan. Sino, que también deberían revisar las propuestas formativas para mejorar "la forma en la que se prepara a los estudiantes para vivir en sociedades que son más complejas y más plurales" ${ }^{18}$ Como así

\footnotetext{
16 TERIGI, F., Conferencia Las cronologías de aprendizaje: un concepto para pensar las trayectorias escolares, Santa Rosa, Ministerio de Cultura y Educación, (2010), P.2

17 Ibid. P. 3

${ }^{18}$ Ibid. P. 4
} 
también pensar en lo que ella denomina "trayectorias reales"19 donde se tienen en cuenta diferentes desafios. Por ejemplo, las ausencias, las transiciones escolares, las relaciones de baja intensidad $^{20}$, la sobre-edad.

La propuesta de la autora es desarrollar un saber pedagógico y un saber didáctico que incrementen la capacidad de los docentes de desarrollar distintas cronologías de aprendizaje ${ }^{21}$, que, a la larga lleven a resultados equivalentes. ${ }^{22}$

Revisar los tipos de saberes que surgen de las experiencias de las escuelas, nos lleva, según Terigi, a repensar y cambiar ciertas políticas educativas.

Porque los niños necesitan que sus derechos educativos se cumplan en sus escuelas. Por lo tanto, la autora sostiene que estos problemas de la política educativa, no deben ser un problema al que deba encontrárseles la vuelta a nivel institucional o individual. ${ }^{23}$

Terigi propone repensar las políticas educativas, los currículums institucionales, y la selección de ejes temáticos. Lo cual no sería solamente definir núcleos temáticos más poderosos, sino que deberían traducirse de manera concreta los diferentes niveles de aprendizaje. $^{24}$

Alicia de Alba nos dice que, en la actualidad, no hay proyectos políticos-sociales capaces de responder a los conflictos que viven las personas. Por lo tanto, esto afecta al campo de los currículums y dificulta el trabajo práctico. ${ }^{25}$ Por lo tanto, comenzamos a pensar

${ }^{19}$ Ibid. P. 4

${ }^{20}$ Ibid.., P. 10

${ }^{21}$ Ibid. P. 18

${ }^{22}$ Ibid. P. 21

${ }^{23}$ Ibid. P. 19

${ }^{24}$ Ibid. P. 22

${ }^{25}$ DE ALBA, A., “Currículum. Crisis, Mito y Perspectivas”, Ed. Miño y Dávila, Buenos Aires, (1995), P. 62 
que debe gestarse, y de hecho en algunos lugares se está gestando, un cambio en las maneras que se entiende la enseñanza y el aprendizaje.

Desde nuestro lugar entendemos el aprendizaje como forma de construir experiencias; y Esteban Rosenzweig, en uno de sus artículos, dice que deberían existir espacios y tiempos donde tanto los profesores como los estudiantes puedan experimentar con el aprender. El autor reflexiona sobre esta cuestión porque se pregunta si aquello que se aprende debería estar determinado. Es decir, se pregunta si se podría aprender en la indeterminación de un espacio, $\mathrm{o}$ en su apertura. ${ }^{26}$ Esto me lleva a pensar en la posibilidad del aprendizaje como una forma de creación. Donde los estudiantes no se encuentren con algo elaborado de antemano, algo que deberían asimilar, así como se les brinda. Sino, pensar en el aprendizaje como un trabajo de laboratorio. Algo más activo.

$\mathrm{Y}$, en este punto es donde encontraremos algunas posibles formas de la modificación que se pretende. En este punto es donde $\mathrm{FcN}$ nos brinda todas sus herramientas para que los profesores $\mathrm{y}$ docentes podamos re-pensarnos y re-pensar nuestras prácticas.

Aquellos que intentamos enseñar filosofía a partir de lo aprendido en $\mathrm{FcN}$ intentaremos transformar el grupo de estudiantes en una comunidad de investigación. Pues coincidimos con Splitter y Sharp que aquellos que participan en una comunidad de indagación construyen y modifican de forma recíproca sus ideas. Enfocan la discusión en una indagación grupal en lugar de dispersarse en direcciones individuales porque el tema sobre el que se discute y se investiga es un tema que despierta su interés. Mantienen el respeto entre ellos $y$ se preocupan y se comprometen con los procedimientos de indagación. Se ayudan a formular preguntas y a

${ }^{26}$ ROSENZWEIG, E., "Espacios abiertos: entre el aprendizaje filosófico y la experimentación con los espacios para la enseñanza de la filosofia", en CERlETTI, A., COUlÓ, A. (Comp.), “Aprendizajes filosóficos. Sujeto, experiencia, infancia”, Ed. Noveduc, Buenos Aires, (2015), P. 96 
ampliar los puntos de vista, sustentan la hipótesis de alguien con un ejemplo o desafían la de otro con un contraejemplo. Dan tiempo y espacio para que las voces tímidas se expresen por sí mismas y las voces agresivas se tornen más reflexivas y consideradas, mostrando que se interesan por la estructura $y_{27}$ los procedimientos de indagación tanto como por su contenido. ${ }^{27}$

La comunidad de indagación permite que los niños se vean como pensadores activos, como descubridores. La comunidad se vuelve una experiencia auténtica para ellos. Pues es un lugar donde lo que ellos piensan, dicen y hacen tiene repercusión en el mundo que los rodea. ${ }^{28}$

Ambos autores aseguran que "la filosofía es una dimensión irremplazable de la educación, así como la educación es una dimensión irremplazable de la filosofía". Y agregan que la filosofía tiene un compromiso con la transformación social en la medida en que contribuye a poner en cuestión, elucidar y entender las injusticias de la sociedad actual, así como también ayuda a pensar las condiciones de posibilidad de superar las mismas.

$\mathrm{Y}$ para los docentes que hacen $\mathrm{FcN}$ y que ejercen sus prácticas desde ese lugar, tuvieron que hacer un ejercicio sobre sí mismos. Es decir, aquellos profesores o docentes que se encontraron con FcN descubrieron que debían hacer una desestructuración en cómo concebían la enseñanza, en cómo habían aprendido que era enseñar, en cómo habían aprendido a ejercer su rol.

Siempre cito a una docente que leí en el libro La escuela pública apuesta al pensamiento, porque ella expresó que la filosofía era morir y nacer de nuevo. Es decir, después de haber vivenciado filosofía con niños, ella considera que la filosofía le había ayudado

${ }^{27}$ SPLITTER, L. y SHARP A., La otra educación. Filosofía con Niños y Comunidad de indagación, Ed. Manantial, Buenos Aires, (1996), P. 38

${ }^{28}$ Splitter, L., Sharp, A., (1996), P.35 
a descubrir todo aquello que debía extinguir, sacar de sí. Se convenció que debía abrirse para conocer de nuevo. Hacer filosofía para ella fue "nacer para conocer nuevamente" 29

Consideramos que el profesor o profesora debería parecerse a lo que Ranciére definía como profesor emancipador ${ }^{30}$; o lo que Laura Agratti, definía como un profesor-productor ${ }^{31}$; o lo que Freire, propone una postura reflexiva-crítica-progresista-transformadora ${ }^{32}$. En palabras de Kohan, deberían volverse infantes. Pero con esto no quiere decir que se infantilicen o se vuelvan niños. Sino, que lo que este autor sostiene es que devenir infante es un situarse intensivamente en el mundo, es un salir del propio lugar para poder situarse en otros lugares, desconocidos, inesperados. Devenir infante seria despojarse del pasado, presente o futuro, porque no

${ }^{29}$ KOHAN, W., OLARIETA, F., La escuela pública apuesta al pensamiento, HomoSapiens, Rosario, (2013), P. 155

${ }^{30}$ RANCIERE, J., El maestro ignorante. Cinco lecciones sobre la emancipación intelectual, Libros del Zorzal, Buenos Aires, (2007), P. 28

31 "Pienso en un profesor que sea capaz de permitirse la distancia que habilite la aparición de la pregunta, y, de este modo, haga efectiva la acción del filosofar y se constituya en un intelectual crítico del propio saber que creía detentar. Ponerse en cuestión para poner en cuestión y convertirse en un sujeto atravesado por la experiencia de la pregunta es una disposición personal e íntima que redundará en su actividad como docente.

Este profesor-productor, es aquel capaz de advertir la diferencia en la repetición, es el profesor-filósofo que encarna la práctica filosófica en el aula, condición de posibilidad para que los alumnos atraviesen la experiencia de la filosofía.

Un profesor que lejos de internalizar en sus alumnos una imagen de maestroverdad sea capaz de desplegar una actitud filosófica que en la experiencia tiene la potencia de provocar la distancia para que surja la pregunta que interpele la verdad recibida de los labios del profesor en clase". (AGRATTI, L., "Repensando, con otras voces, los sentidos del filosofar", en La escuela pública apuesta al pensamiento, KOHAN, W., OLARIETA, F., (Coordinadores), Ediciones Homosapiens, Rosario, (2013), P. 228)

32 FREIRE, P., La educación como práctica de la libertad, Siglo XXI Editores, Buenos Aires, (2008), P. 19 
habría una temporalidad cronológica, sino que habría un tiempo de pensamiento con geografía, intensidad y dirección construida. ${ }^{33}$

En el libro La escuela pública apuesta al pensamiento, Kohan sostiene que "devenir infante sería formarse para des-formarse, para transformarse, para disponerse, para ponerse de otro modo frente al mundo, para des-aprender, para preguntarse por lo que nadie se pregunta, para problematizar por medio del pensamiento" (Kohan, W., Olarieta, F., 2013-P. 157)

Por eso, creemos que se invita a los docentes a que se vuelvan infantes, para que desarrollen la capacidad de transformar ciertos lugares propios en los que se habían formado. Pero esto, consideramos, no apunta, a que los mismos se despojen totalmente de su trayectoria y vivencias; sino que se está pensando en que el docente pueda "nacer de nuevo", que realice una re-significación de sus prácticas pedagógicas. Se piensa en aquellos docentes que comienzan a formarse como coordinadores de comunidades, y que reconstruyen la experiencia del aula viviendo las comunidades y abandonando esquemas pre-establecidos que a veces se los sostiene en el tiempo y en la práctica como incuestionables. Se está pensando en aquellos docentes que buscan "no repetir, que se permiten ver y verse; que se permiten escuchar, ${ }^{\text {. }}$.

Aquellos docentes que reflexionamos críticamente acerca de la enseñanza de la filosofía, coincidimos en que se debe abordar la asignatura desde una perspectiva diferente. Es decir, no se planea proponer clases meramente académicas y expositivas; sino que, a partir de nuestra concepción de la filosofía, proponemos realizar clases activas, de intercambio, con trabajos grupales y debates que enriquezcan tanto a los estudiantes como a la/los docentes.

La filosofía es una práctica reflexiva, que desarrolla la creatividad, el aspecto ético y valorativo de las personas. Por lo tanto, se

${ }^{33}$ KOHAN, W., Infancia, política y pensamiento, Ed. Cit., P. 95 34 KOHAN, W., OLARIETA, F., Op.cit. P. 193 
pretenderá propiciar el espacio para que los estudiantes se enfrenten a sus experiencias, y a la realidad, y problematicen aquellas nociones que parecen impuestas o que se naturalizan.

Consideramos necesario que los estudiantes se sientan involucrados en las clases, por eso y para eso, debemos prestar atención a la forma en la que abordaremos los temas propuestos. Si deseamos que ellos vivan la filosofía, y adquieran el interés por acercarse de manera problematizadora sobre ciertos temas y puedan fundamentar sus ideas, debemos innovar en el encuentro que les proponemos con la filosofía.

Se debe construir un espacio donde, junto a los estudiantes, podamos encontrarnos con la filosofía desde varias perspectivas. Y también donde el aula funcione como laboratorio de ideas, de prácticas, de proyectos, de acciones. Deseando que el grupo de estudiantes logre conformar una comunidad de investigación e indagación.

En palabras de Litwin, diremos que

la enseñanza requiere que se provoque a los estudiantes para que realicen diferentes actividades con el objeto de aprender, dada la certeza de que los mismos aprenden mejor cuando participan activamente de la organización y búsqueda de relaciones entre la información nueva y la ya conocida.

\section{¿Qué es Filosofía para Niños?}

Filosofía para Niños fue la respuesta de Lipman a lo que él consideró que eran las necesidades de la educación norteamericana a finales de los años 60. Pues la educación tradicional no parecía responder a las necesidades de los estudiantes ni a los de la sociedad. Por esta razón, Lipman pensó que se debía enseñar a los niños a razonar, pero los cursos de lógica se ofrecían demasiado tarde, y no se enseñaba a los alumnos cómo aplicar las habilidades 
lógicas a las distintas disciplinas. Por lo tanto, su proyecto pretendía ofrecer un enriquecimiento conceptual y cultural al mismo tiempo que podría mejorar las habilidades de comprensión, de análisis y de resolución de problemas. ${ }^{35}$

Echeverría, asegura que se podría pensar el programa lipmaniano como dos vertientes que se complementan. Por un lado, como una propuesta educativa, y por el otro, como un proyecto filosófico. Carla Barreras ${ }^{36}$, por su parte, agrega que, en tanto propuesta educativa en este programa se puede hallar la influencia de aquellos pensadores que no estaban convencidos con los resultados del sistema educativo tradicional; por ejemplo Mead, Vigotsky ${ }^{37}$, Dewey o Piaget, entre otros. ${ }^{38}$ Pues, de sus teorías, Lipman extrajo las características formales y metodológicas que fundamentan el currículum de Filosofía para Niños.

Pero además de fomentar, estimular y contribuir al descubrimiento del sentido de las experiencias vitales de los niños, Echeverría

35 LIPMAN, M.-GAZZARD, A., Philosophy for Children: Where we are now...(I), Thinking, vol. 6, $\mathrm{n}^{\circ} 4$, Special Center Supplement, Upper Montclair, 1987, p. 53

${ }^{36}$ BARRIENTOS RASTROJO, J., Op.Cit., P. 92

${ }^{37}$ La perspectiva histórico-cultural desarrollada por Vigotsky es una de las bases fundamentales del constructivismo, el cual se basa en la idea de que el niño debería dejar de ser un recipiente pasivo en su proceso de aprendizaje y convertirse en un actor dinámico dentro de su propio proceso de enseñanzaaprendizaje. (ECHEVERRÍA, E., Filosofia para Niños, Edit. Aula Nueva, México, (2006), P. 32)

38 El pragmatismo es otra de las corrientes que ejerce influencia en Lipman. Es una corriente heterogénea y diversa que plantea al pensamiento como un arma o una herramienta para lograr una acción más efectiva. Dos de los principales representantes son Charles Pierce y William James, quienes rechazaban la idea de que debía haber una serie de certezas o fundamentos esenciales a partir de los cuales se desarrollaría el conocimiento. En cambio, dan importancia a los efectos, las consecuencias y las prácticas en las que se ven envueltos los humanos y ven al pragmatismo como un método para pensar con claridad. (ECHEVERRÍA, E., Op.Cit.,, P. 26s) 
asegura que, el proyecto de Lipman pretende incidir en la capacidad democrática de los individuos, contribuyendo al desarrollo de las habilidades de pensamiento ${ }^{39}$, el trabajo en valores y la creación de actitudes. ${ }^{40}$

Si leemos a Lipman, nos dirá que el objetivo de Filosofía para Niños no es enseñarles fillosofía a los niños, sino hacer que los niños filosofen, que piensen mejor por sí mismos; que puedan reflexionar por sí mismos sobre el sentido y el alcance de los grandes conceptos filosóficos ${ }^{41}$.

Lipman nos dice esto porque para él su paradigma, su ejemplo a seguir en esta forma de entender la filosofía, es Sócrates ${ }^{42}$, quien

${ }^{39}$ Las habilidades de pensamiento al desarrollarse, van a permitir pensar mejor, es decir, pensar de manera lógica, sin contradicciones y se podrán detectar argumentos falaces o erróneos. Además, el desarrollo de las habilidades de pensamiento permite a las personas defenderse de la manipulación de los medios de comunicación o de ciertos discursos políticos. (ECHEVERRÍA, E., Op.Cit., P. 57)

${ }^{40}$ BARRIENTOS RASTROJO, J., Op.Cit.,., P.92

${ }^{41}$ Dentro de FpN, existirían dos tipos de filosofía. Por un lado, la filosofía que aparecería como una instancia privilegiada en orden a desarrollar un buen pensar, ya que resulta concebida como la única disciplina que reflexiona sobre el propio pensar, sobre sus criterios, y sobre la evaluación de éstos; como la disciplina que está conectada con este pensamiento en tanto proceso y en tanto contenido. Y por el otro, una filosofía como autocorrección del pensamiento reflexivo que resulta un componente determinante en cuanto a cuestiones de juicio y evaluación regida por reglas y principios lógicos, habilidades de pensamiento, etc. (SHARP, A., Op.Cit., P. 130)

42 "El modelo para hacer filosofía es la gran figura solitaria de Sócrates, para quien la filosofía no era una adquisición, ni una profesión, sino una forma de vida. Lo que Sócrates nos enseña no es a saber filosofía, ni aplicarla, sino a practicarla. Nos desafía a reconocer que la filosofía es, en cuanto un hacer, en cuanto una forma de vida, algo que cualquiera de nosotros puede imitar. (..) Lo que Sócrates subrayaba era el continuo ejercicio de la investigación filosófica siguiendo el razonamiento hasta donde nos lleve. (...) Los niños deben ejercitarse en la discusión de los conceptos que ellos se toman en serio. (...) Sócrates no establece en ningún lugar que no se pueda hacer filosofía con gente 
proponía un camino de investigación, de examen e interrogación a partir del diálogo. Es por esto que se podría sostener, junto a Splitter y Sharp, que Filosofía para Niños consta de dos componentes básicos; la comunidad de indagación ${ }^{43}$ y el diálogo filosófico.

Tanto Lipman como estos autores, sostienen que la actividad filosófica "sólo es posible en un contexto de intercambio y contraste de opiniones y razones para una construcción compartida de sentido y un ejercicio de metacognición" 44 .

A su vez, el diálogo filosófico y la comunidad de indagación están interconectados con el desarrollo de las habilidades de pensamiento y de razonamiento; las cuales, según Lipman, deben considerarse como un conjunto de instrumentos indispensables en el proceso educativo.

Sumiacher, a partir de su lectura de Echeverría, asegura que Lipman produjo una ruptura con el modo habitual de concebir la relación infancia y filosofía, y que otorgó un especial valor a las capacidades pensantes de niños y niñas que suelen ser poco valoradas en los contextos escolares. Lipman no estaba de acuerdo

de diferentes edades, porque hacer filosofía no es asunto de edades, sino de capacidad para reflexionar escrupulosa y valientemente sobre lo que a uno le parece importante". (LIPMAN, M., SHARP, A., OSCAYAN, F., La filosofia en el aula, Ediciones de la Torre, Madrid, 1992, P. 30s)

43 "La frase "comunidad de indagación" viene de la tradición pragmatista, en especial de Pierce, Mead y Dewey. Referida inicialmente a la indagación científica entre adultos. Sin embargo, esta noción es central para un entendimiento de Filosofía para Niños" (SPLITTER, L. y SHARP A., La otra educación. Filosofía con Niños y Comunidad de indagación, Ed. Manantial, Buenos Aires, P. 50)

La comunidad de indagación es la metodología básica propuesta por Lipman y Sharp, consistente en trabajar sentados en círculo, intercambiando formas recíprocas de comunicación, en las que las ideas pasan a compartirse y a ser accesibles e importantes para otros. (SPLITTER, L. y SHARP, A., Op.Cit., P. 56)

${ }^{44}$ BARRIENTOS RASTROJO, Op. Cit., Ed. Cit., P. 97 
con el paradigma piagetiano ${ }^{45}$, aún vigente en ese entonces, donde se asumía que los niños se hallarían con la posibilidad de hacer "razonamientos formales" a partir de los doce años o más; y la filosofía era pensada, para muchos, como un estudio exclusivamente académico. ${ }^{46}$

Lipman generó un giro epistemológico respecto de los objetivos que la filosofía tiene como materia escolar. Sus trabajos van desde la teorización más abstracta y formal sobre el lugar del juicio o las habilidades de pensamiento, a las novelas filosóficas para niños, desarrollando aspectos práctico-metodológicos-pedagógicos. En su tesina ${ }^{47}$, Sumiacher nos cuenta que las habilidades cognitivas fueron centro de interés académico e influenciaron sobre el movimiento de pensamiento crítico a partir de 1950 con Benjamin Bloom, entre otros. La memoria fue considerada en ese momento, como el primer escalón. Luego seguiría la comprensión, el análisis, la capacidad de síntesis y en la cima de la pirámide, la evaluación. La acumulación de conocimiento ya no era tan importante para los

${ }^{45}$ Esto es así, según Echeverría, porque las escuelas normalistas y las facultades de pedagogía han tomado y enseñado a Piaget de manera superficial, incompleta y mal informada. Lo cual trajo como consecuencia que muchos maestros no enseñen ciertos temas porque "los niños no lo entenderían ya que todavía están en la etapa de operaciones concretas"; y que la filosofía, y el hecho de pretender que los niños hagan filosofía, se vea como imposible dada la incapacidad de los niños para pensar de manera abstracta y de trabajar con pensamiento hipotéticodeductivo. Sin embargo, Piaget constituye un soporte teórico para FpN. Lipman tomó su descripción de los cambios cualitativos en el niño con la llegada de las operaciones formales como un elemento importante para introducir algunos de los aspectos más importantes de la lógica formal aristotélica. (ECHEVERRÍA, E., Op. Cit., Ed. Cit., P. 35)

46 SUMIACHER D’ANGELO, D., “John Dewey y la Filosofía para Niños de Matthew Lipman", Artículo publicado en: HARADA, E. (Comp.) (México, 2012), La filosofia de Matthew Lipman y la Educación: Perspectivas desde México, UNAM.

47 SUMIACHER, D., Nuevos enfoques para y desde Filosofía para Niños, Tesina, (2008), P.35 
pedagogos, por lo tanto, el pensamiento evaluativo había ascendido en las teorizaciones cognitivas. Para ese entonces, las ideas piagetianas tenían un gran auge, y los teóricos consideraban que los niños no podían enfrentarse a ideas abstractas por la etapa biológica en la que se hallaban.

En la tesina escrita por David Sumiacher encontramos que, "a finales de los años 70 se reconsidera el paradigma piagetiano, y se retoma a autores como Vygotsky ${ }^{48}$, por lo cual surgen movimientos como el de la lógica informal o el constructivismo, distanciándose de la lógica dura y se busca una teoría que sirviese para el lenguaje cotidiano". Además agrega que se podría decir que Ryle, Wittgenstein y Austin, entre otros, prepararon el camino para que estos movimientos pudiesen surgir. ${ }^{49}$

El aporte sustantivo y específico de Lipman consiste en el papel que atribuye a la filosofía en la experiencia educativa y en su idea

48 “Para Vigotsky el lenguaje es un medio de comunicación social íntimamente ligado con el desarrollo de la inteligencia. Sostiene que el desarrollo del pensamiento no es posible sin lenguaje. Los procesos psicológicos superiores aparecerían en las personas en forma interpersonal, y luego se desarrollarían de manera intrapersonal. Esto quiere decir que el bebé desarrolla sus procesos psicológicos desde el momento en que la madre comienza a interactuar con él. Aprende a darle significado a su propia experiencia a través de la forma en que otras personas cercanas interactúan con él.

Una vez que se hace consciente de la función del lenguaje para dar sentido a su experiencia y entender el ambiente que lo rodea, el niño es capaz de internalizar el lenguaje que ha aprendido y de pensar internamente.

Otra noción importante es la de zona de desarrollo potencial. Esta zona sería el espacio entre lo que una persona puede aprender por sí sola, y lo que puede desarrollar con la interacción de otros. Reuven Feurestein, creador del Programa de Enriquecimiento Instrumental, llama grado de modificabilidad cognitiva a esta situación. Pues todas las personas presentan modificabilidad cognitiva, pero todas son capaces de avanzar más en el desempeño y desarrollo intelectual con la mediación de otros". (ECHEVERRÍA, E., Op.Cit., P. 34)

${ }^{49}$ SUMIACHER, D., Op.Cit., P. 36 
revolucionaria de que los niños son aptos para practicar filosofía tan pronto como comienza su educación institucionalizada. ${ }^{50}$

Kohan y Waksman sostienen que Lipman comparte con Dewey la idea de que la educación debe ser un proceso de reconstrucción de la experiencia del estudiante y, a diferencia de éste, apuntala a la filosofía, y no a la ciencia, como forma de investigación principal para ello. La filosofía resulta para Lipman una disciplina que prepara a los niños para pensar en las otras disciplinas y así, les otorga herramientas que les permitirían encontrar sentido y problematicidad en su experiencia. Lipman amplía el carácter teórico de la actividad filosófica atribuido por Dewey, hacia una práctica dadora de sentido de la experiencia pedagógica desde los niveles más temprano de la escolaridad.

Lipman escribió una reconstrucción de la historia de la filosofía occidental en forma de novelas filosóficas. Estas novelas fueron pensadas como lectura de jóvenes adolescentes, o niños; es por esto que, las escribió incluyendo diálogos entre jóvenes o niños esperando que los lectores se sintiesen identificados con los personajes. También escribió manuales para docentes que no tuviesen formación en filosofía. En estos manuales, Lipman propone ejercicios y planes de discusión sobre las ideas principales contenidas en las novelas. ${ }^{51}$

\section{Elementos de Filosofía para/con Niños}

Consideramos que uno de los desafíos de los profesores y profesoras, como sostiene Cuellar (2016), es convertir el aula en un espacio filosófico. Y para lograrlo, los profesoras y profesoras,

${ }^{50}$ KOHAN, W., WAKSMAN, V., Filosofía con Niños. Aportes para el trabajo en clase, Ediciones Novedades Educativas, Buenos Aires, (2005), P. 29

${ }^{51}$ KOHAN, W., WAKSMAN, V., Op.Cit., P. 39 
podrían servirse de los siguientes elementos, por llamarlos de alguna manera.

En Filosofía para Niños existen dos componentes básicos; la comunidad de indagación ${ }^{52}$ y el diálogo filosófico. La comunidad de indagación es la metodología básica propuesta por Lipman y Sharp, consistente en trabajar sentados en círculo, intercambiando formas recíprocas de comunicación, en las que las ideas pasan a compartirse y a ser accesibles e importantes para otros. ${ }^{53}$

La presencia de los otros es significativa en el trabajo reflexivo sobre el pensamiento. Por eso es tan importante trabajar en comunidad de indagación. Splitter y Sharp sostienen que la comunidad de indagación

Evoca un espíritu de cooperación, de cuidado, de confianza, de seguridad y de un sentido de propósito común y de indagación que genera una forma de práctica autocorrectiva dictada por la necesidad de transformar lo que es intrigante, problemático, confuso, ambiguo o fragmentario en alguna clase de totalidad unificadora que satisfaga a aquellos que están involucrados y que culmine, aunque sea de modo tentativo, en el juicio ${ }^{54}$

En el mismo texto, los autores agregan que aquellos que participan en una comunidad de indagación construyen y modifican de forma recíproca sus ideas. Enfocan la discusión en una indagación grupal en lugar de dispersarse en direcciones individuales porque el tema sobre el que se discute y se investiga es un tema que despierta su interés. Mantienen el respeto entre ellos y se preocupan y se

52 "La frase "comunidad de indagación" viene de la tradición pragmatista, en especial de Pierce, Mead y Dewey. Referida inicialmente a la indagación científica entre adultos. Sin embargo, esta noción es central para un entendimiento de Filosofía para Niños" (SPLITTER, L. y SHARP A., La otra educación. Filosofía con Niños y Comunidad de indagación, Ed. Manantial, BUENOS Aires, P. 50)

${ }^{53}$ SPLITTER, L. y SHARP, A., Op.Cit., P. 56

${ }^{54}$ SPLITTER, L. y SHARP A., Op.Cit., P. 37. 
comprometen con los procedimientos de indagación. Se ayudan a formular preguntas y a ampliar los puntos de vista, sustentan la hipótesis de alguien con un ejemplo o desafían la de otro con un contraejemplo. Dan tiempo y espacio para que las voces tímidas se expresen por sí mismas y las voces agresivas se tornen más reflexivas y consideradas, mostrando que se interesan por la estructura y los procedimientos de indagación tanto como por su contenido.

En toda comunidad resulta importante el diálogo filosófico, el cual se distingue de una mera conversación ${ }^{56}$, o de un intercambio de opiniones, o de un diálogo científico, o de una terapia de grupo, por la búsqueda y el contraste de razones. Según Carla Carreras, en un diálogo filosófico "la finalidad principal no es convencer al otro, sino llegar a encontrar uno mismo las razones que le ayuden a fundamentar mejor sus propias opiniones y creencias" ${ }^{, 57}$.

La discusión filosófica es una experiencia intelectual compartida en la que un grupo de individuos se transforma en una comunidad donde sus ideas y pensamientos pasan a compartirse; y donde se comprende que tanto las ideas personales como la de los demás integrantes resultan igualmente importantes.

Splitter y Sharp, en su libro La otra educación proponen algunas condiciones como necesarias y suficientes para considerar a la conversación como diálogo.

1) La conversación es estructurada por su concentración en un tópico o cuestión que es problemático o polémico. ${ }^{58}$

55 SPLITTER y SHARP, Op.Cit., P. 38

56 “...la distinción entre las formas corrientes de conversación, como resultado de la interacción entre individuos, por un lado, y la conversación estructurada o diálogo, por otra". (SPLITTER y SHARP, Op.Cit., P. 57s)

57 BARRIENTOS RASTROJO, J., Op.Cit., P. 96

58 "No se intentan excluir las discusiones en las que los estudiantes ejercitan sus habilidades creativas o expresivas aun cuando no pueda haber ningún problema claro guiando la discusión (...) parte de lo que es problemático en una discusión o indagación, en realidad puede ser identificar un problema o los problemas". 
2) La conversación es autorregulación o autocorrección. Sus participantes están preparados para cuestionar los puntos de vista y razones expuestos por otros, y para reformular su propia posición en respuesta a los cuestionamientos $\mathrm{y}$ contraejemplos provenientes del grupo.

3) La conversación tiene una estructura igualitaria. Los participantes muestran que, a los efectos del diálogo, se valoran a sí mismos y a los otros de igual modo, independientemente de donde estén situados en relación con un punto de vista particular.

4) La conversación es guiada por los intereses mutuos de sus miembros. En la comunidad de indagación son los participantes quienes establecen la agenda y determinan los procedimientos para tratar los asuntos en cuestión.

En el mismo texto de Splitter y Sharp encontramos que el diálogo, entendido como la conversación característica de la comunidad de indagación, no es un debate, ya que este último implica necesariamente participantes que toman diferentes, y a veces opuestos, puntos de vista.

A pesar de que muchas veces algunos participantes pueden sentir rechazo o enojo frente al cuestionamiento de sus creencias o valores, estos autores sostienen que es importante que se animen a ser más abiertos, ya que uno de los objetivos del diálogo es aumentar la comprensión de quienes toman parte en él; y para ello, la mayoría de las veces, deben vivenciar procesos en donde tendrán que volver y reflexionar sobre sus supuestos y así reconstruir sus propios puntos de vista.

Otro elemento importante es el rol del coordinador. Es decir, hasta que la comunidad de indagación adquiera cierta madurez, el profesor o profesora deberá cumplir cierto rol. Deberá realizar sobre sí un ejercicio de transformación, de de-construcción.

(SPLITTER y SHARP, Op.Cit., P. 59) 
El coordinador o coordinadora debe poseer y desarrollar apertura para escuchar lo que los chicos dicen sin apresurarse a interpretar; paciencia para que el proceso de pensamiento pueda desarrollarse con el tiempo propio de cada chico y de cada grupo en particular; sensibilidad al contexto para evaluar cuándo conviene intervenir y de qué modo; y atracción por los problemas, por las preguntas complejas, por los procesos de investigación. ${ }^{59}$

El coordinador o coordinadora también debe poner a disposición de los chicos un objeto estimulante o provocador de la reflexión. Debe estar atento/a a que cada uno se exprese con claridad, a que cada uno sea escuchado y comprendido por los demás. También debe cuidar que las relaciones entre todos favorezcan la cooperación y no la competencia. ${ }^{60} \mathrm{Y}$ pondrá a disposición de los chicos las herramientas argumentativas que favorecerán la clarificación del pensamiento y su formulación. ${ }^{61}$

Sharp y Splitter sostienen que la comunidad de diálogo es un espacio pedagógico que puede ser tomado como alternativa metodológica por aquellos maestros que buscan formas, estrategias, herramientas, para formar niños y adolescentes críticos, reflexivos, que piensen por sí mismos; que desarrollen un espíritu democrático, de tolerancia, una actitud de entendimiento y respeto a la diversidad y una capacidad crítica. ${ }^{62}$

En Filosofía con Niños encontramos que, además de estos elementos, a veces se trabaja con clases especiales, donde no se leen las novelas de Lipman, ni se aplica la metodología de las comunidades de investigación al estilo lipmaniano, sino, que se

\footnotetext{
${ }^{59}$ SANTIAGO, G., Filosofía, niños, escuela. Trabajar por un encuentro intenso, Paidós Educador, Buenos Aires, (2006), P. 256

60 SANTIAGO, G., ¿Es posible trabajar la reflexión en la escuela?, Ed. Biblos, Buenos Aires, (2010), P. 19s

61 SANTIAGO, G., Filosofía, Niños, Escuela, Ed. Cit., (2006), P. 61

62 SHARP, A., SPLITTER, Op.Cit., P.
} 
trabaja con actividades como las que propone Proyecto Noria ${ }^{63} \mathrm{o}$ Gustavo Santiago. Pero las mismas pueden utilizarse como recurso para trabajar cualquier problemática filosófica.

De hecho, según lo que se pretenda trabajar, en lo que se conoce como prácticas filosóficas o Filosofía aplicada, los profesores y profesoras encontrarán diferentes propuestas. Algunas, como sostiene Sumiacher, serán concernientes más al trabajo lingüístico argumental, otras introducirán elementos de la lúdica, algunas pondrán el acento en las habilidades de pensamiento, y otras destacarán el valor de la razón experiencial; algunas se enfocarán en el aspecto crítico, y otras en la dimensión creativa del pensamiento. ${ }^{64}$

Pero podemos asegurar que, cualquiera de los elementos mencionados aquí, deberían ser apropiados como herramientas por aquellos profesores y profesoras que busquen convertirse en transformadores activos de su propia actividad, ya que estos elementos les permitirán esclarecer las condiciones ideológicas y materiales que operan en sus prácticas docentes. Y que también deseen promover la formación de estudiantes con actitud reflexiva, crítica, sujetos autónomos, y libres, pero con conciencia social.

Cerletti y Kohan nos dirán que sólo quien pueda reflexionar activamente sobre sus prácticas, estará en condiciones de trascender los diseños de ejecución e implementación burocratizados, empleando formas pedagógicas que traten a los estudiantes como agentes críticos capaces de cuestionar la legitimidad de un hecho o una argumentación, proponiendo $\mathrm{y}$

63 "Proyecto Noria es una propuesta educativa para niños de entre 3 y 11 años, que nació a mediado de los años 90 resultado de la unión de esfuerzos entre Irene de Puig (España) y Angélica Sátiro (Brasil)". (DE PUIG, I., SÁTIRO, A., Jugar a penar con niños y niñas de 4 a 5 años, Ed. Octaedro, Barcelona, 2011, P. 13) 64 SUMIACHER, D., Op.Cit., P.3 
trabajando sus problemas e intereses a partir del diálogo y el intercambio reflexivo. ${ }^{65}$

\section{Conclusión}

Haremos la conclusión de este escrito como una invitación a seguir pensando la propuesta presentada. Y con la intención de haber aportado, desde nuestra experiencia, otra forma de entender la enseñanza de la filosofía, pero también, presentando uno de los muchos ámbitos en los que podemos poner en práctica Filosofía con Niños.

La filosofía sería ese camino que uno emprende y que ayuda a encender el pensamiento. Un pensamiento en el que nos involucramos, en el que se toman posiciones ante diferentes cuestiones que resultan importantes en nuestras vidas.

Creemos en que la filosofía puede ser esa herramienta, esa práctica, que ayude y permita que nos constituyamos en personas capaces de pensar la realidad en general, y nuestras realidades particulares y subjetivas, y que ese pensar a su vez sirva para estar en mejores condiciones de decidir nuestra relación con el mundo, creo que resulta importante dirigir la mirada analítica a la enseñanza y a la práctica de la filosofía. Pues entre otras cosas, es importante pensar el lugar que la filosofía desempeña en el diseño curricular porque eso permitiría comprender las posibilidades y limitaciones, y así se podrían conseguir, proponer, nuevas relaciones pedagógicas.

Como sostienen Kohan y Cerletti, se debe abordar el problema del sentido de la filosofía en la escuela de "hoy", desde una perspectiva filosófica. Y esto supone, tener presente las condiciones actuales de

${ }^{65}$ CERletTI, A., KOHAN, W., La Filosofía en el aula, La UBA y los Profesores Secundarios. Oficina de publicaciones de C. B. C., Buenos Aires, 1996, P. 27 
producción de un saber filosófico y las posibilidades de su enseñanza. Los alumnos están inmersos en un espacio sociocultural posmoderno, cuando, las escuelas a las que asisten, en su mayoría, son estructural e históricamente, modernas. ${ }^{66}$

La escuela moderna tiene por objeto formar los espíritus, y tiende, según Condorcet, a 'borrar el límite entre la porción grosera y la porción iluminada del género humano'; los alumnos posmodernos, retraducen este objetivo emancipador en programa arcaico de sujeción y confunden, en un mismo rechazo de autoridad la disciplina y la transmisión, el maestro que instruye, y el amo de domina ${ }^{67}$

Desde la perspectiva de ambos autores, la aproximación filosófica al problema de la enseñanza de la filosofía obliga a tematizar el rol y el lugar del profesor, en tanto pensador activo y promotor de la reflexión compartida con los alumnos interlocutores. Es por esto que algunos filósofos consideran que la práctica filosófica en el aula debería partir de los intereses e inquietudes que surgen de las propias experiencias para volverse reflexión y puesta en cuestión de esa experiencia.

La discusión filosófica en las aulas deviene problematización y cuestionamiento compartido de aquellos valores, saberes, prácticas y creencias que subyacen a la cotidianeidad ${ }^{68}$

La filosofía en la escuela adquiere una re-significacion. Deja de ser algo abstracto, imposible de entender, para ser recibida como una nueva forma de encuentro con el otro, con los otros. La filosofía en la escuela comienza a ser esa nueva forma de ver las cosas;

${ }^{66}$ KOHAN, W., CERLETTI, A., Op. Cit., Ed. Cit., P. 47

${ }^{67}$ (Finkielkraut, A., La derrota del pensamiento, (1987), Barcelona, Anagrama, 1990, P. 131) Cita extraída del libro La filosofía en la escuela, KOHAN, W., CERLETTI, A., La UBA y los Profesores Secundarios, Buenos Aires, (1996), P. 12

${ }^{68}$ KOHAN, W., CERLETTI, A., Op.Cit., P. 86 
comienza a ser esa nueva manera de ver cosas que antes no se veían, o que se veían distinto, es empezar a mirar "con otros ojos". $\mathrm{Y}$ finalizamos este escrito asegurando que seguiremos pensando filosóficamente acerca de la enseñanza y de los vínculos entre los docentes, profesores y estudiantes. Porque una educación emancipadora es posible y necesaria. Porque pensamos que la filosofía, como dice Derrida, citado en el mismo texto, excede las instituciones.

Creemos necesario educar filosóficamente en instituciones que respeten las experiencias y que puedan acompañar y funcionar como nexo, como lazo conductor entre los niños y ciertas formas de acceder y habitar el mundo.

El desafío de quien quiere educar acompañando la experiencia reside en que cuenta con frágiles gestos en espacios donde rigen normas, y en que su gesto es siempre una invitación ${ }^{69}$

\section{Bibliografía}

AGRATTI, L., "Repensando, con otras voces, los sentidos del filosofar", en La escuela pública apuesta al pensamiento, KOHAN, W., OLARIETA, F., (Coordinadores), 2013, Rosario, Ediciones Homosapiens

BARRIENTOS RASTROJO, J., Filosofía para Niños $y$ capacitación democrática freiriana, Edita Liber Factory, Madrid, 2013

CERLETTI, A. (Comp.), 2009, La enseñanza de la filosofía en

\footnotetext{
${ }^{69}$ FREIXAS, J., "Tensiones filosóficas en la educación: entre las demandas acreditadoras y la inquietud de la infancia", Artículo publicado en el libro de CERletTI, A., COUlO, A. (comp.), 2015, Aprendizajes filosóficos. Sujeto, experiencia e infancia, Buenos Aires, Edic. Novedades Educativas, P.36
} 
perspectiva, Buenos Aires, Ed. Eudeba

CERLETTI, A., 2015, Didáctica aleatoria de la filosofía, dialéctica del aprendizaje filosófico, en Didácticas de la filosofía. Entre enseñar y aprender a filosofar, CERLETTI, A., COULÓ A. (Orgs.), Buenos Aires, Ed. Noveduc

CERLETTI, A., KOHAN, W., 1996, La Filosofía en la escuela, Buenos Aires, La UBA y los Profesores Secundarios. Oficina de publicaciones de C. B. C.

DE ALBA, A., Currículum. Crisis, Mito y Perspectivas, 1995, Buenos Aires, Ed. Miño y Dávila

DE PUIG, I., SÁTIRO, A., Jugar a penar con niños y niñas de 4 a 5 años, Ed. Octaedro, Barcelona, 2011

ECHEVERRÍA, E., Filosofía para Niños, Edit. Aula Nueva, México, 2006.

FREIRE, P., 2008, La educación como práctica de la libertad, Buenos Aires, Siglo XXI Editores

FREIXAS, J., "Tensiones filosóficas en la educación: entre las demandas acreditadoras y la inquietud de la infancia", Artículo publicado en el libro de CERLETTI, A., COULO, A. (comp.), 2015, Aprendizajes filosóficos. Sujeto, experiencia e infancia, Buenos Aires, Edic. Novedades Educativas

GARCÍA MORENTE, M., 1980, Lecciones preliminares de Filosofia, Versión digital, México, Editorial Porrúa

HEIDEGGER, M., 2004, ¿Qué es la filosofía?, Barcelona, Ed. Herder

JASPERS, K., 1953, La Filosofia desde el punto de vista de la existencia, México, Fondo de cultura económica

KOHAN, W., OLARIETA, F., 2013, La escuela pública apuesta al pensamiento, Rosario, HomoSapiens

LIPMAN, M.-GAZZARD, A., Philosophy for Children: Where we are now...(I), Thinking, vol. $6, \mathrm{n}^{\circ} 4$, Special Center Supplement, Upper Montclair, 1987 
LIPMAN, M., SHARP, A., OSCAYAN, F., La filosofía en el aula, Ediciones de la Torre, Madrid, 1992

NESPRÍAS, J., "Enseñanza de la filosofía y contextos 'difíciles': límites, posibilidades, desafíos (una aproximación desde El maestro ignorante, de Jacque Ranciére", en Cerletti, A. (Comp.), 2009, La enseñanza de la filosofía en perspectiva, Buenos Aires, Ed. Eudeba ONFRAY, M., "El deseo de ser un volcán", en Cerletti, A. (Comp.), 2009, La enseñanza de la filosofía en perspectiva, Buenos Aires, Ed. Eudeba

RANCIÉRE, J., 2003, El maestro ignorante, Barcelona, Editorial Laertes

RANCIERE, J., 2007, El maestro ignorante. Cinco lecciones sobre la emancipación intelectual, Buenos Aires, Libros del Zorzal ROSENZWEIG, E., "Espacios abiertos: entre el aprendizaje filosófico y la experimentación con los espacios para la enseñanza de la filosofía", en CERLETTI, A., COULÓ, A. (Comp.), 2015, Aprendizajes filosóficos. Sujeto, experiencia, infancia, Buenos Aires, Ed. Noveduc

SUMIACHER D'ANGELO, D., “John Dewey y la Filosofía para Niños de Matthew Lipman", Artículo publicado en: Harada, E. (Comp.) (México, 2012), La filosofía de Matthew Lipman y la Educación: Perspectivas desde México, UNAM.

SUMIACHER, D., Nuevos enfoques para y desde Filosofia para Niños, Tesina, 2008

SPLITTER, L. y SHARP A., 1996, La otra educación. Filosofía con Niños y Comunidad de indagación, Buenos Aires, Ed. Manantial

TERIGI, F., 2010, Conferencia Las cronologías de aprendizaje: un concepto para pensar las trayectorias escolares, Santa Rosa, Ministerio de Cultura y Educación

UICIH, F., "Las decisiones filosóficas detrás de las decisiones didácticas: consejos para repensar la planificación de clases de filosofía”, en CERLETTI, A., COULÓ, A., 2015, Didácticas de 
Filosofía. Entre enseñar y aprender a filosofar, Buenos Aires, Ediciones Novedades Educativas 